\title{
HIGH POWER RF TEST FACILITY AT THE SNS*
}

\author{
Y. Kang, D. Anderson, I. E. Campisi, M. S. Champion, M. Crofford, R. Fuja, P. Gurd, S.M. S. Hasan, K. Kasemir, \\ M. McCarthy, D. Stout, J. Tang, A. Vassioutchenko, M. Wezensky \\ SNS Project, Oak Ridge National Laboratory, Oak Ridge, TN \\ G.K. Davis, M. Drury, T. Powers, M. Stirbet, Thomas Jefferson National Accelerator Facility (TJNAF), VA
}

\begin{abstract}
RF Test Facility (RFTF) has been constructed to support present and future needs in testing, processing and conditioning of various high power RF components of normal conducting and superconducting systems at the SNS. The facility is expected to have additional subsystems that are needed for complete superconducting RF (SRF) testing and processing. A full capacity high voltage converter modulator (HVCM) with $11 \mathrm{MW}$ peak power at $8 \%$ duty cycle has been installed for driving one or two klystron RF amplifiers. The waveguides are completed in WR-2100 and WR-1150 for the $402.5 \mathrm{MHz}$ and $805 \mathrm{MHz}$ klystrons being used in the SNS. The 805 MHz system has been used for RF processing the coaxial fundamental power couplers (FPCs) for the SNS superconducting linac (SCL) [1]. The high power RF system can be reconfigured or modified for various tests and conditioning processes along with the neighboring cryo-plant.
\end{abstract}

\section{INTRODUCTION}

Construction and installation of the accelerators in the SNS project are entering the final stage. A fully capable RFTF was included in the initial project planning and has been constructed during the construction of the SNS linac. The RFTF was expected to be a flexible RF facility and a dedicated test-bed for various room-temperature and cryogenic high power RF components: cavities, couplers, etc. The facility is equipped with a shielded cave for RFprocessing the superconducting cavities in cryomodules and room temperature accelerating structures. The SRF test setup will include clean rooms and vertical dewars that can be used for complete SRF processing. The SNS uses $402.5 \mathrm{MHz}$ and $805 \mathrm{MHz}$ HPRF systems in its linac; present transmitter setups can accommodate available pulsed RF klystron amplifiers that are the same types being used in the SNS linac. The high power RF system is equipped with an $8 \%$ duty, $11 \mathrm{MW}$ peak pulsed HVCM with two klystron sockets that can support two RF generators with output power up to $2.5 \mathrm{MW}$ at $402.5 \mathrm{MHz}$ and $5 \mathrm{MW}$ at $805 \mathrm{MHz}$ [2]. At this point, temporarily, a $550 \mathrm{~kW} 805 \mathrm{MHz}$ klystron is installed for the testing needed to support the SNS project and other high power coupler processing tasks. After completion of construction and installation of the SNS linac, various spares and new development of high power RF components will need to be processed, conditioned, and tested to their full power

\footnotetext{
* SNS is managed by UT-Battelle, LLC, under contract DE-AC05-00OR22725 for the U.S. Department of Energy.
}

specifications for the SNS project and for other scientific projects if desired. Presently, a LabView based computer controlled RF processing setup with interlock system has been being used. For efficient RF processing, an EPICS (Experimental Physics and Industrial Control System) based RF conditioning and processing control system independent to the accelerator system operation is needed. A full-featured EPICS based system is being developed and tested [3]. A computer-controlled baking system is also being used to prepare the components before RF processing.

\section{SRF FACILITY}

The RFTF shares a building with the cryogenic plant. This has the advantage of future testing of cold cryomodules in the facility. The current SNS cleanroom located inside the RFTF consists of portable, or softwall, cleanrooms housed within a $20^{\prime}$ by $30^{\prime}$ filtered tent. Laminar flow benches are used within the portable cleanrooms for drying, assembly, and baking, while a portion of the larger tent houses ultrasonic cleaning stations. An ultra-pure water system (300 gallon capacity) supports cleaning and rinsing. Bench assembly is particulate-free - 0 at 0.3 microns. The assembly portable cleanroom is Class 10 (without activity). The baking cleanroom and overall tent are Class 100.

A permanent facility is planned based on the requirements to maintain, repair and test existing cryomodules and to support production of upgrade cryomodules. Office space/cubicles would be removed from the existing RF building, and the following systems added:

- 45' by $30^{\prime}$ Cleanroom divided into Class 10,000 and Class 100 areas

- $12^{\prime}$ by $20^{\prime}$ Chemistry room with an acid scrubber system

- 15' by $30^{\prime}$ Cryomodule assembly area (plastic curtained area)

- An above-ground vertical test assembly (VTA) area with two shielded dewars

- A sub-atmospheric Helium pump for VTA and test cave operation

Helium would be supplied by the Central Helium Liquifier (CHL) and vented. Planning also is underway for a separate testing/backup refrigeration system.

\section{HIGH POWER RF CAPABILITY}

The test facility has one HVCM power supply and two HV tanks each supporting one klystron with complete waveguide plumbing; two klystrons can run at the same 
time. The WR-1150 and WR-2100 waveguide systems are presently installed for the same types of klystrons being used for the SNS. New klystrons can also be tested at the RFTF; three spare 402.5 MHz, 2.5 MW klystrons from Thales Electron Devices for the SNS drift tube linac (DTL) are to be factory certified and site acceptance tested on the SNS RFTF test stand with the engineer from the manufacturer. There exists a flexibility to mount klystrons for other frequencies if necessary. The planned configuration with the socket and waveguide is to operate one $805 \mathrm{MHz} 5 \mathrm{MW}$ tube and one $402.5 \mathrm{MHz}, 2.5 \mathrm{MW}$ tube [4]. Presently, an $805 \mathrm{MHz} 550 \mathrm{~kW}$ CPI tube is installed for the RF processing of the FPCs with a beam stick (klystron diode) in the $402 \mathrm{MHz}$ high voltage tank to balance the HVCM load. The RF Test Facility has been used for HVCM power supply testing and RF conditioning more than half of the JLAB built 81 FPCs. The FPCs were conditioned with traveling and fullyreflected waves up to $650 \mathrm{~kW}$ peak forward power in $1 \mathrm{msec}, 60 \mathrm{~Hz}$ pulses.

Circulators are used to isolate the klystron from the devices under processing that is terminated with either a well matched water load or a fully reflecting adjustable short. These two types of loads have been used during the conditioning of the FPCs for the superconducting cavities of the SNS. Directional couplers are used to measure the input and output powers before and after the device setup under processing. Since most high power RF components used in accelerator systems work under vacuum, it is desirable to have a pair of devices to be conditioned with a connecting waveguide (or cavity) that is evacuated. Two FPCs with a rectangular connecting waveguide has been used in the RF conditioning. Figure 1 shows the klystron, HVCM and high power RF distribution system in the RFTF.

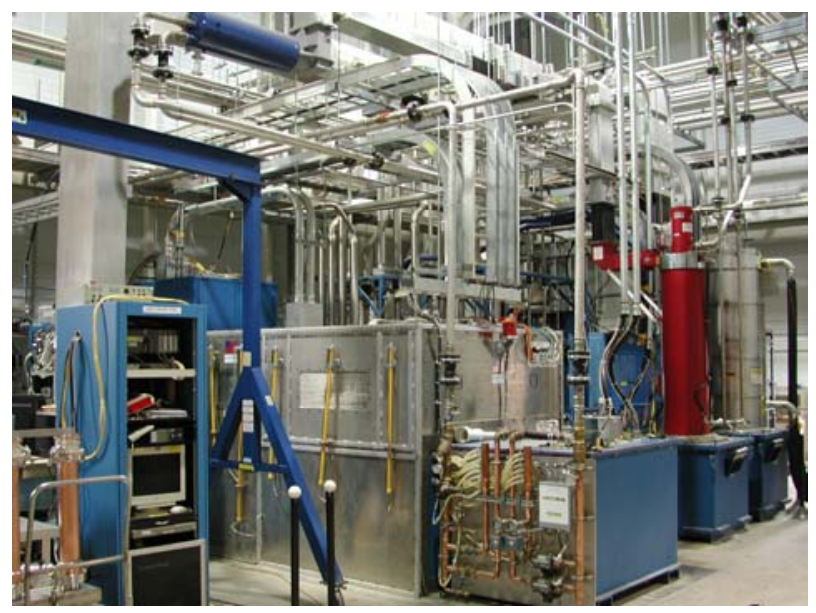

Figure. 1: The layout of the high power RF system in the RFTF.

\section{RF CONDITIONING CONTROL SYSTEM}

Presently a control system for RF power and vacuum using LabView is used for high power RF processing of the FPCs of the SNS superconducting cavities. Operation and control of the HVCM and the transmitter with klystrons are done in the EPICS based system. EPICS implementation of the RF control systems has been found to be more desirable and is being tested. This work is to have a completely integrated RF processing control system for improved long-term operation of the test facility. Figure 2 shows the simplified block diagram of the RF processing control system.

Some instruments used are: MKS vacuum gauge controllers and cold cathode gauges (CCG), vacuum pumping cart, arc detector, RF power meters, RF signal generator. A fast RF switch interlock, and transmitter control for klystron amplifiers and high voltage converter modulator are implemented in the PLC control and EPICS systems. Other control hardware include Vacuum IOC (Input Output Controller), Klystron IOC, RF Power IOC, Allen Bradley Programmable Logic Controller (PLC) where the IOCs are based on VXI and control software is EPICS.

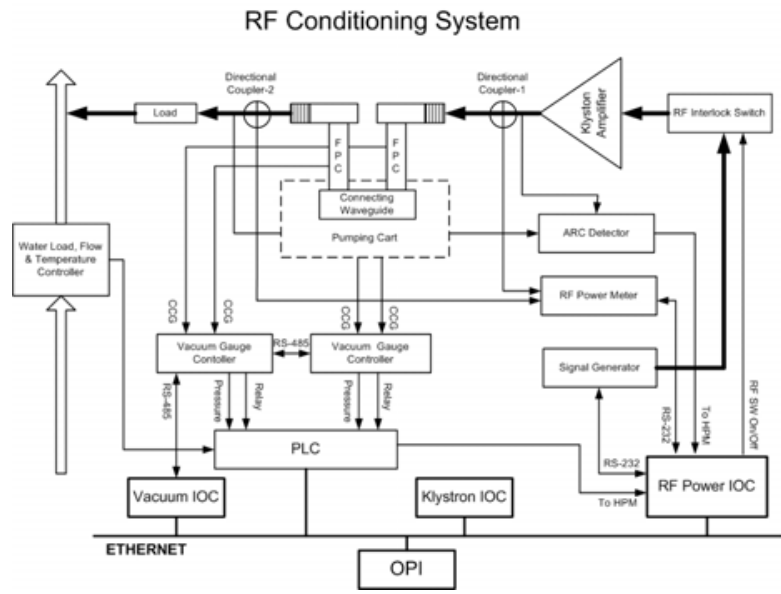

Figure. 2: Overall block diagram of the RF conditioning system.

It is necessary to monitor vacuum quality, any signs of arcing, and forward and reflected RF power levels for the whole RF conditioning process of high power vacuum components such as cavities, couplers and windows. The amplified RF from the klystron amplifier will be transmitted to the devices under processing through the waveguide transmission line. Between the klystron and a coupler there is a directional coupler to monitor the power signal. In the following discussion, couplers are used as the devices under conditioning. The RF power is transmitted to another coupler through a connecting waveguide and finally reaches the termination that is a water load for traveling wave processing or an adjustable short for standing wave processing. Another directional coupler is used between the load and coupler for monitoring the RF matching at the termination. Two RF power meters each with two channels are connected to the RF power IOC through RS-232 to measure the forward and reflected power levels at the input and the output ports of the couplers.

Two cold cathode gauges (CCG) are used to monitor the vacuum inside each coupler. These four gauges are connected to two MKS vacuum gauge controllers (VGC). 
Every VGC has five modules - an analog module, which gives the pressure reading in raw analog format, a relay module, which gives the relay status, a communication module helps to communicate with other device, and two CCG controller modules measure the pressure. The analog modules and relay modules are directly connected to the PLC to supply the pressure and relay status information, respectively. If vacuum pressure crosses the predefined upper limit then the PLC will send a RF off request signal to the high power module (HPM) of the RF power IOC.

The IOC will shut down the RF interlock switch within a few microseconds. So, the PLC is used for continuously monitoring the vacuum level and other auxiliary systems like water load, flow and temperature conditions. Communication modules are also connected with the vacuum IOC through RS-485 for controlling the VGC's remotely. RS-485 is a bit slower than the raw analog and digital communication. But if the vacuum pressure goes above the upper limit then the RF needs to be shut down as fast as possible: so the raw analog and relay signals are directly connected to the PLC for taking the necessary actions. The RS-485 communication is used to configure the VGCs remotely.

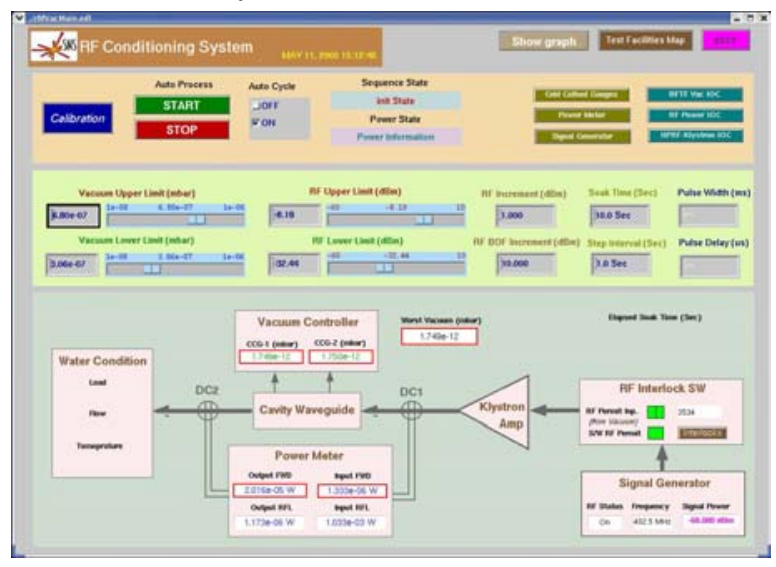

Figure. 3: Main control screen of the EPICS based RF conditioning system.

A signal generator is connected to the RF power IOC through RS-232 connection. The output of this signal generator goes to the RF interlock switch and delivered to the driver amplifier. If the IOC gives the permission to pass the signal then the switch passes the signal to the driver for klystron amplifier. A separate IOC controls the klystron amplifier and the modulator. An arc detector is used on each coupler to detect the arc during the RF conditioning process and it is connected to the HPM of the RF power IOC. RF power IOC shuts down the switch to interlock as soon as any arc occurs. So the HPM is the main module to get all the signals for controlling the RF interlock switch.
Since all IOCs and PLC are connected through Ethernet, it is possible to control and monitor the conditioning process from the accelerator control room and anywhere else if desired. EPICS is to be used in the whole RF conditioning control system for more robust and flexible, but simple process control and monitoring. Main operator interface (OPI) screen was designed in such a way that the operator can observe the status of any instrument, set the parameters, control every step of the conditioning process, and archive the data and results. The extensible display manager (EDM) screen of the designed OPI is shown in Figure 3.

\section{CONCLUSION}

The RFTF has been used to complete the RF processing of about half of the 81 fundamental power couplers for the SNS linac. All couplers have been installed on the superconducting cavities in the cryomodules. Baking the ceramic windows and coaxial structures used in the couplers has also been done at the RFTF. Assembling and testing the air-side coupler transitions also used on the SCL cryomodules have been completed at the RFTF. Preparation of the RF test station for RF processing of other normal conducting accelerating cavity components is under way: spare components such as couplers for the RFQ and windows for the drift tube linac will need to be conditioned. The RF test facility at the SNS is becoming a full featured high power RF Test facility that is capable of supporting the work need to be done with both superconducting and room temperature systems for the SNS and possibly for other scientific projects in the accelerator community.

\section{ACKNOWLEDGEMENT}

The authors are thankful to other SNS personnel who worked hard to have the RFTF set up and run with least problems. The authors are also thankful to JLAB personnel who helped to RF process the fundamental power couplers for the SNS superconducting linac successfully.

\section{REFERENCES}

[1] M. Stirbet et al, "RF Conditioning and Testing of Fundamental Power Couplers for SNS Superconducting Cavity Production," PAC 2005

[2] M. McCarthy, et Al. "Spllation Neutron Source High Power RF system Installation and Commissioning Progress," PAC 2005

[3] S.M.S. Hasan, et al. "EPICS Based High Power RF Conditioning Control System for the SNS Accelerator RF Test Facility," submitted to ICALEPCS-2005, Geneva, Switzerland, October 2005.

[4] Karen Young et al, "5MW $805 \mathrm{MHz}$ SNS RF System Experience," PAC 2005 